\title{
Correction to: Estimation of satellite position, clock and phase bias corrections
}

\author{
Patrick Henkel $^{1} \cdot$ Dimitrios Psychas $^{1} \cdot$ Christoph Günther $^{1,2} \cdot$ Urs Hugentobler $^{1}$
}

Published online: 6 July 2018

c) Springer-Verlag GmbH Germany, part of Springer Nature 2018

\section{Correction to: Journal of Geodesy} https://doi.org/10.1007/s00190-018-1146-y

On pages 1, 2 and 11, "bernese GNSS software" should be replaced by "Bernese GNSS Software".

In Fig. 8(c), the label (c) is missing at the upper-left corner.

Figure 8(e) was wrongly selected and should be replaced by the new Figure 8(e) as shown below.
In Fig. 9(a), the label (a) is missing at the upper-left corner. In Fig. 9(e), the x-axis is incomplete, i.e. "Time [epochs]" should be extended to "Time [epochs of $100 \mathrm{~s}$ ]" to be consistent with the other subfigures. The attached version of Fig. 9(e) has the corrected $\mathrm{x}$-axis.

The original article can be found online at https://doi.org/10.1007/ s00190-018-1146-y.

$凶$ Patrick Henkel

patrick.henkel@tum.de

Christoph Günther

christoph.guenther@dlr.de

1 Technische Universität München, Munich, Germany

2 German Aerospace Center (DLR), Oberpfaffenhofen, Germany 

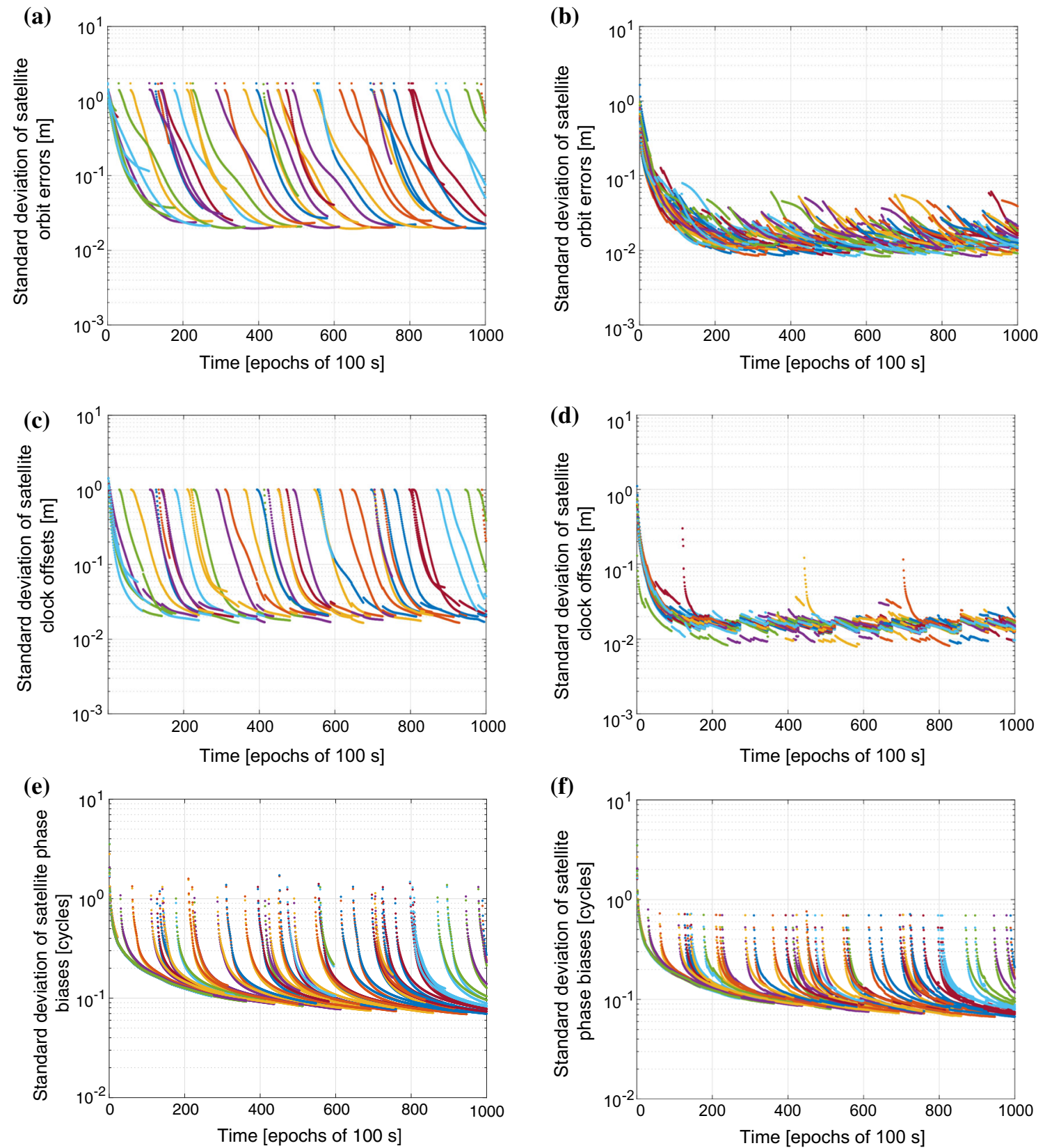

Fig. 8 Benefit in precision of multi-cluster estimation of satellite position corrections, satellite clock offsets and phase biases with float ambiguities. Each colour represents a different satellite. a Single-cluster satellite position corrections. b Multi-cluster satellite position correc-

tions. c Single-cluster satellite clock offsets. d Multi-cluster satellite clock offsets. e Single-cluster satellite phase biases. f Multi-cluster satellite phase biases 

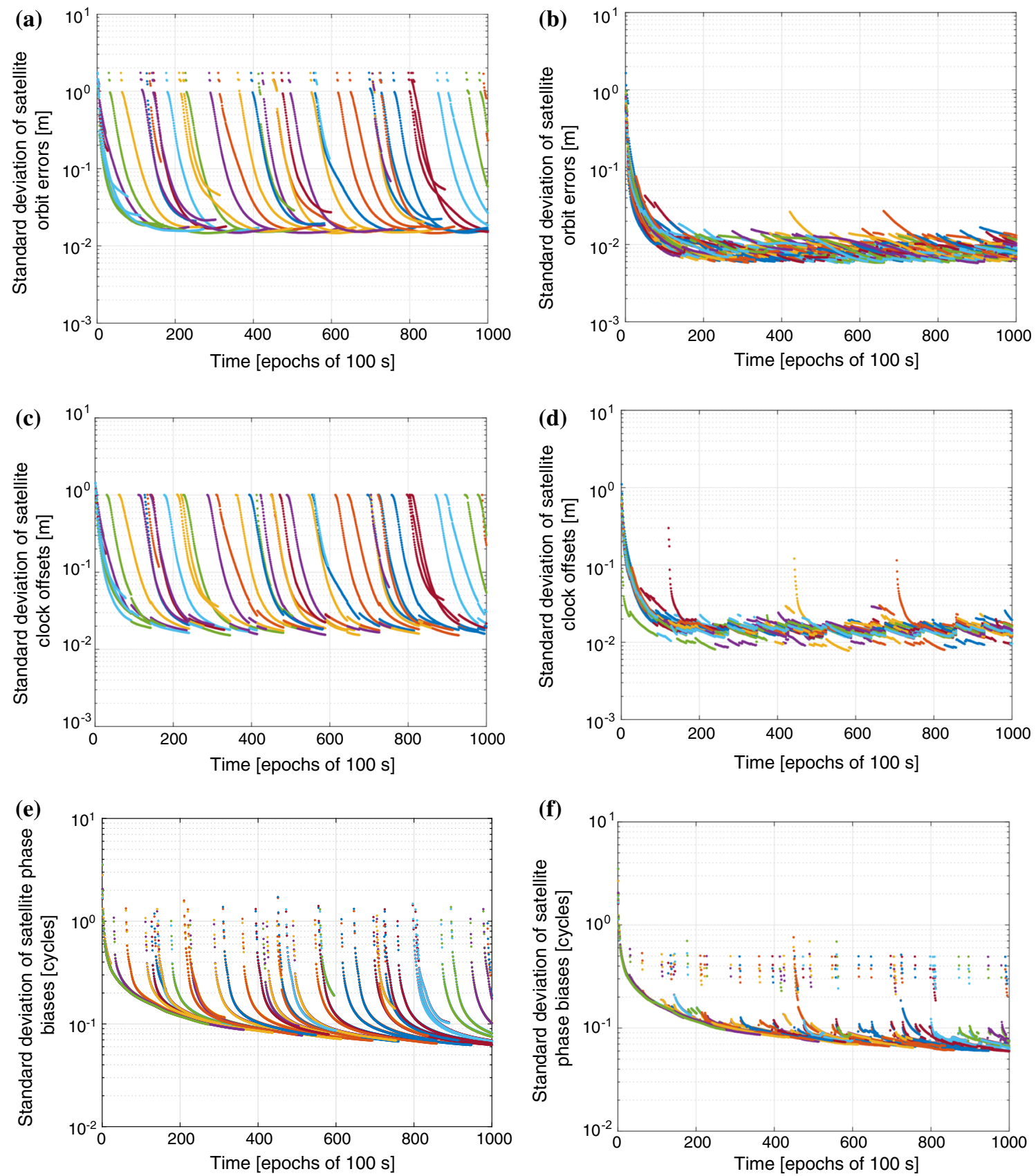

Fig. 9 Benefit in precision of multi-cluster estimation of satellite position corrections, satellite clock offsets and phase biases with fixed ambiguities. Each colour represents a different satellite. a Single-cluster satellite position corrections. b Multi-cluster satellite position correc-

tions. c Single-cluster satellite clock offsets. d Multi-cluster satellite clock offsets. e Single-cluster satellite phase biases. f Multi-cluster satellite phase biases 